A $R$ R T I I C C U L L O O D $\begin{array}{lllllllll}R & E & F & L & E & X & I & O & N\end{array}$

\title{
Profesionales de trabajo social EN LA BUROCRACIA DE LAS NACIONES UNIDAS $^{1}$
}

\author{
THE SOCIAL WORKERS IN THE UNITED NATIONS BUREAUCRACY \\ SYSTEM
}

Por: Claudia Bermúdez*

* Trabajadora Social (Universidad del Valle) magíster en Educación Popular (Universida del Valle), Candidata a Doctora en Ciencias Sociales, Universidad Nacional General Sarmiento Buenos Aires

Argentina
1 El artículo se realiza en el marco de la investigación doctoral titulada "Construcción de la experiencia intersubjetiva del trabajo de trabajadores sociales" actualmente en curso. Fecha de inicio: febrero 2014. La investigación cuenta con el respaldo de la Universidad de Valle
Recibido: 18 de febrero de 2018 - Aprobado: 28 de mayo de 2018

\section{RESUMEN}

El artículo se propone visibilizar y describir la participación de organizaciones de Trabajo Social y de trabajadores sociales como parte de la burocracia del sistema de las Naciones Unidas y en ese sentido muestra, por un lado la configuración de la disciplina/profesión en el marco de unas relaciones globales que la hicieron posible y por otro, la manera como llegó a América Latina. El periodo de tiempo para el análisis comprende dos décadas que van desde el surgimiento de las Naciones Unidas en el año 1945, teniendo en cuenta que desde ese momento organizaciones de profesionales de Trabajo Social se vincularon de manera activa como organismos no gubernamentales, hasta la década de los sesenta, dada la ruptura que colectivos de trabajadores sociales en Latinoamérica plantearon con el perfil de profesionales de Trabajo Social creados desde la ONU. Un eje transversal para el análisis, lo constituye el reconocer a este sistema intergubernamental como un escenario proveedor de empleo para trabajadores sociales y la influencia de esa condición en los desarrollos de la disciplina/profesión.

Palabras clave: Trabajo Social; Trabajadores Sociales, Reconceptualización, Trabajo Social Latinoamericano, Desarrollo de la comunidad, Burocracia, Naciones Unidas.

\begin{abstract}
The article aims to visualize and describe the participation of Social Work organizations and social workers as part of the bureaucracy of the United Nations system and in that sense shows the configuration of the discipline / profession within the framework of global relations that made it possible and the way it arrived in Latin America. The period of time for the analysis includes two decades that go from the emergence of the United Nations in the year 1945, taking into account that from that moment organizations of professionals of Social Work were linked of active way like nongovernmental organisms, until the decade of the sixties, given the break that groups of social workers in Latin America raised with the profile of Social Work professionals created from the UN. A transversal axis for the analysis is recognizing this intergovernmental system as a scenario providing employment for social workers and the influence of that condition on the development of the discipline / profession.
\end{abstract}

Keywords: Social Work; Social Workers; Reconceptualization; Latin American Social Work; community development, Bureaucracy, United Nations 


\section{Introducción}

Tra participación de los científicos sociales como parte de la burocracia del Sistema de las Naciones Unidas representa una importante referencia Jen torno a la manera como se ejerce un control sobre las actividades de los mismos (Wallerstein, 2006); las ciencias sociales en este marco no parecen ejercerse como una actividad autónoma, sino como una actividad que desde la subordinación laboral se ajusta a las demandas institucionales que requieren un "conocimiento práctico". El presente artículo tiene como objetivo describir la participación de profesionales del Trabajo Social como parte de la base burocrática de las Naciones Unidas y las implicaciones que dicha participación trajo en el ejercicio profesional, entendiendo a la ONU como un escenario proveedor de empleo que ejerce un control directo sobre las formas de actuación y sobre el cambio social que se propone. El período de referencia para el análisis corresponde a las dos primeras décadas de las Naciones Unidas (1946-1966); considerando este periodo clave en dos sentidos, por un lado porque la vinculación de organizaciones de profesionales del trabajo social a esta entidad se realizó casi desde su mismo surgimiento y en segundo lugar porque fue en la década del sesenta en América Latina, cuando se planteó una ruptura frente a la manera como se había orientado la formación profesional desde allí. Del mismo modo, hay que señalar que profesionales del Trabajo Social hicieron parte del amplio cuerpo burocrático de dicha entidad.

Para cumplir este objetivo el artículo está organizado en cuatro grandes apartados. El primero de ellos describe la metodología seguida en la que se señala que se trató de un trabajo de análisis documental con una perspectiva histórica. El segundo apartado, presenta la participación de organizaciones de Trabajo Social en las Naciones Unidas y de trabajadores sociales como empleados y parte de la base burocrática de dicha organización. En el tercer apartado se puntualiza en las responsabilidades, cargos y funciones que desempeñaron, su influencia directa en las decisiones y perspectivas de la entidad y finalmente la manera como fueron recibidas las ideas que sobre el Trabajo Social construyó las Naciones Unidas, en Latinoamérica.

\section{Metodología}

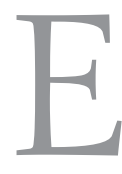

l trabajo es resultado de una revisión y análisis documental de textos institucionales que permitieron ubicar al Trabajo Social en una red de relaciones de carácter global. Desde ese punto de vista el análisis más que en los contenidos desarrollados desde la disciplina/profesión está puesto en la articulación a redes internacionales y su influencia en la configuración del 
2 Mediante el artículo 71 de la carta de las Naciones Unidas, se definió que "El Consejo Económico y Social podrá hacer arreglos adecuados para celebrar consultas con organizaciones no gubernamentales que se ocupen en asuntos de la competencia del Consejo. Podrán hacerse dichos arreglos con organizaciones internacionales". De esta manera y ajustándose al protocolo y a las normas las ONG interesadas en vincularse al sistema debían realizar una solicitud y presentarla ante el comité de $\mathrm{ONC}$ de dicho Consejo -ECOSOC- La solicitud era ampliamente estudiada y luego, en sesiones ordinarias el Consejo definía si aprobaba o no su vinculación a su vez que asignaba una clasificación.

3 Para el 2014 dentro de las Organizaciones de Trabajadores Sociales que participan activamente del Consejo Económico y Social -ECOSOC- de las Naciones Unidas como Organismos no Gubernamentales se encuentran: la Federación Internacional de Trabajadores Social (FITS), la Asociación Internacional de Escuelas de Trabajo Social (IASSW), el Servicio Social Internacional y la Sociedad de Amigos del Servicio Social. Del mismo modo asociaciones más locales como por ejemplo el Consejo de Servicio Social de Hong Kong, el Mauritus Council Social Service y asociaciones religiosas como la agencia de Servicio Social de la Iglesia Protestante Alemana, la Unión Católica Internacional por el Servicio Social, así como el Comité Católico Internacional de Enfermeras, Médicos y Trabajadores Sociales. También han participado otras organizaciones que no se ha sostenido en el tiempo como por ejemplo la Asociación Profesional de Trabajadores Sociales y Prácticas del Desarro-
Trabajo Social como producto de múltiples interconexiones e intersecciones. Si bien el análisis lo sitúo en una perspectiva histórica, no es un trabajo histórico y si bien acudí a fuentes primarias, principalmente las actas de las asambleas de las Naciones Unidas de las primeras décadas (1946-1966), fueron relevantes las fuentes secundarias en la medida que me permitieron acercarme al ámbito de lo relacional; dentro de las fuentes secundarias retomé textos institucionales y los textos publicados por organizaciones internacionales de Trabajo Social. Los documentos fueron sometidos a tres tipos de lectura: Lectura descriptiva, lectura interpretativa y una lectura comparativa

\section{Resultados}

\section{Organizaciones de trabajadores sociales y las Naciones Unidas}

Tempranamente Organizaciones Internacionales de Trabajo Social, principalmente de países del norte, mostraron su interés en vincularse al Sistema de las Naciones Unidas en calidad de Organismos no Gubernamentales -ONG- a través del Consejo Económico y Social -ECOSOC-. Las Naciones Unidas desde su surgimiento definieron para las ONG un estatus consultivo² ${ }^{2}$ Eso significó que dichas ONG tenían la posibilidad de asistir, intervenir, presentar sus opiniones y puntos de vista frente a temas de debate pero no tenían posibilidad de votar. En otras palabras, significó la posibilidad de ser escuchado y de enviar declaraciones. Este estatus en la actualidad se mantiene.

Las primeras organizaciones de Trabajo Social que presentaron solicitud y obtuvieron el estatus consultivo fueron el Comité Internacional de Escuelas de Servicio Social y el Servicio Social Internacional en el año 1947³. Las Naciones Unidas también parecían mostrar un especial interés en el Trabajo Social. En efecto, en el año 1946 por ejemplo, la Asamblea General expresaba la relevancia de contar con un número apropiado de peritos de Servicio Social que asesorara y pusiera en práctica métodos en cualquier ramo del bienestar social para el diseño de programas eficaces. La ONU consideraba que estos profesionales debían encargarse en países del "tercer mundo" del apoyo a programas de desarrollo y en países devastados por la guerra de programas de reconstrucción. Fue así como profesionales del Trabajo Social entraron a hacer parte de distintas misiones de expertos del organismo. 
En los años 1950, 1956 y 1958 la ONU también realizó los primeros estudios internacionales sobre formación en Trabajo Social ${ }^{4}$. En todos los casos los resultados parecían arrojar "escasez" de un personal capacitado y entrenado en este ámbito ${ }^{5}$. A partir de estos estudios, las Naciones Unidas definieron como tarea inmediata la promoción y creación de Escuelas de Trabajo Social en diferentes regiones del mundo, para lo cual brindaron su asesoría y recomendaron a los gobiernos la vinculación de este tipo de profesionales a sus programas. Esta difusión se apoyó en un volumen importante de publicaciones en distintos idiomas sobre Trabajo Social (Resolución 58 (I), 1946).

Para dar cumplimiento a tal tarea en el año 1951 (Resolución 390-XIII1951) el ECOSOC pidió al secretario general formular, en cooperación con organizaciones intergubernamentales y no gubernamentales, unas normas mínimas relativas a la creación de programas de formación en Trabajo Social y solicitó que cada cuatro años se rindiera un informe que señalara los avances en esta materia. El secretario también solicitó una guía revisada de Escuelas de Trabajo Social.

De otro lado, y a partir del informe sobre la situación social en el mundo realizado en el año $1952^{6}$, el ECOSOC se propuso como objetivo la búsqueda de su pronta solución. Ello, desde su punto de vista, solo era posible con la puesta en marcha de programas de acción práctica, -como por ejemplo el diseño de métodos de administración de servicios sociales-, y la asesoría a los gobiernos para la creación de programas de formación en Servicio Social.

Para la Federación Internacional de Trabajadores Sociales -FITS-, todo esto lo que muestra es que el estatus consultivo de las organizaciones de Trabajo Social en modo alguno han representado una participación pasiva. Por el contrario, creen que dar a conocer sus declaraciones y puntos de vista en escenarios como la asamblea, les ha permitido consolidarse como referente importante para el direccionamiento de los temas que allí se debaten. Para la FITS ello puede verse claramente reflejado en la manera como la ONU, incorporó acciones con individuos, grupos y comunidades (definidos en ocasiones como niveles de intervención del trabajo social) (Mastrangelo de pamphilis, 2003) y también en la promoción que comenzó a hacer del Trabajo Social en distintas regiones del mundo. Si bien la FITS resalta que cuenta con una agenda global propia, también reconoce su contribución al desarrollo de la agenda de la ONU, principalmente en lo que respecta a las acciones del ECOSOC y de la oficina de asuntos sociales. (Cronin, Sakina Mama, Mbugua, \& Mouravieff-Apostol, 2006). llo de la India, la Asociación de Trabajadores Sociales de Kuwait, la Escuela Universitaria de Trabajo Social de Columbia, entre otras. 4 Dichos estudios fueron titulados "Training for social work, an international survey" (1950). El tercer estudio contó con traducción al español

5 Esta "escasez" ya la venía señalando desde años atrás Eleaonor Roosevelt, esposa de Franklin Delano Roosevelt, - presidente de los Estados Unidos entre1933-1945-. Eleaonor ocupó un lugar relevante en el ECOSOC, principalmente en lo que respecta a la redacción de la Declaración Universal de los Derechos Humanos en el año 1948. Ella conocía de años atrás a Jane Addams y el Hull House, lo que le permitió tener un referente del campo de acción del trabajo social. Por ello no solo lo exaltaba de manera permanente sino que también consideraba que era necesario expandir su entrenamiento en el mundo. https://www2.gwu. ed u / erpapers myday/displaydoc. c fm ? y $=1943$ \& $\mathrm{f}=\mathrm{md} 056494$

6 El objetivo de este informe fue la identificación de problemas sociales presentes en los países "insuficientemente desarrollados". Los temas foco de atención fueron las condiciones sanitarias, alimentación, habitación, instrucción, condiciones de trabajo y empleo, circunstancias que influyen en el nivel de vida, nivel de renta y bienestar, nacimientos, defunciones, morbilidad (paludismo, tuberculosis) y otros. 
En el año 1954 la Asamblea General de la ONU ya expresaba su satisfacción frente a los programas de asistencia técnica en servicio social que habían emprendido. Afirmaba que constituían una de sus más fructuosas realizaciones y por ello invitó a la implementación de acciones con los gobiernos para que el programa adquiriera una mayor eficacia (Resolución, 831-IX, 1954). En el año 1955, tal asamblea reafirmó su confianza en la asistencia técnica al considerar que se trataba de un medio para promover el progreso económico y social y en 1959, felicitó la eficacia con la que se había llevado a cabo hasta el momento.

Lo que puede observarse hasta aquí es que buena parte de la "confianza" de las Naciones Unidas en el trabajo de organizaciones de Trabajo Social estaba dada por los atributos asignados al mismo. Por ello es común encontrar en las fuentes documentales adjetivos que se refieren al trabajo social como "rápido", "eficaz", "práctico" lo que de entrada resultaba especialmente atractivo para el desarrollo de su agenda institucional. Así por ejemplo lo manifestaban en el año 1955, cuando se incorporó el método de desarrollo de la comunidad como parte de las acciones del personal de Servicio Social (resolución del ECOSOC 585-XX). Allí se afirmaba que, "teniendo en cuenta la apremiante necesidad que tienen muchos países de aumentar rápidamente el número de trabajadores sociales con formación especializada y seleccionado por sus afinidades y aptitudes personales y su comprensión de las posibilidades y necesidades de las personas con quienes habrá de trabajar, hay que insistir en las ventajas que ofrece no solo formar y emplear trabajadores sociales profesionales sino también formar y emplear en ciertas cosas trabajadores polivalentes y auxiliares" (Negrita mía). Lo que se puede advertir es que profesionales del Trabajo Social de alguna manera contaban con legitimidad para hacer parte de la burocracia de las Naciones Unidas.

La confianza de la ONU en el Trabajo Social fue tan grande que incluso estableció el día del Trabajo Social, el cual se celebra anualmente, hasta la actualidad. En este contexto, ¿De qué se ocupó el Trabajo Social en las Naciones Unidas? ¿Qué tipo de tensiones debieron enfrentar los profesionales?

\section{Profesionales de trabajo social en las Naciones Unidas}

Pese a que he venido señalando el interés reiterado y sostenido que parece haber tenido la ONU en el trabajo social, Wolfe (1982) afirmó que la presencia de profesionales de las áreas sociales no estuvo exenta de tensiones y disputas en su interior, principalmente relacionadas con dominios del saber. 
En efecto, en torno al informe preliminar sobre la situación social en el mundo de 1952, el autor identificó la existencia de una serie de controversias en las que se podían ver reflejadas claramente dos posiciones. En la primera de ellas situó a los economistas quienes, según el autor, se asumían a sí mismos como la versión "autorizada" en materia de desarrollo económico haciendo uso de herramientas como la econometría y medición cuantitativa. Al parecer, este colectivo de profesionales no veía razones para incorporar lo social como una variable necesaria para el logro del desarrollo.

La segunda posición agrupó a profesionales del Servicio Social, quienes proponían que lo social debía ser direccionado tomando como modelo la experiencia que por décadas venían realizando Organizaciones de la Caridad de los Estados Unidos. Wolfe señala que si bien no asumían la postura de jerarquía del saber propia de los economistas, "daban por descontado que las formas de acción social con las que se identificaban eran derechos humanos esenciales, y que sus normas y técnicas, con adaptaciones menores, serían adecuadas para todo el mundo". Por ello el autor afirma que aun cuando representaban la incorporación de lo social a la perspectiva del desarrollo, "eran tan insensibles como los economistas a las cuestiones de cambio social estructural y relaciones de poder" (Wolfe, op.cit).

Al parecer, en medio de estas tensiones derivadas por los dominios de saber, las posibilidades de desplegar y avanzar en debates teóricos parecían nulas. De hecho Wolfe afirmó que los debates en ese sentido eran evadidos y no se asumían con profundidad, en parte porque la ONU insistía en la necesidad de enfatizar en asuntos prácticos de modo que la teoría quedaba relegada a un segundo plano. De este modo, "el proyecto de enfoque unificado no logró realizar una elección fundamentada entre las teorías ya existentes ni tampoco elaborar una teoría original. Además de los evidentes obstáculos de falta de tiempo y de divergencias (disciplinarias y de otro carácter) en el seno del equipo, se veía inhibido por el prejuicio contra la teorización que mostraban los auspiciadores institucionales del proyecto. Según dicho prejuicio, la argumentación teórica produce divisiones; además, de acuerdo con lo que suele repetirse en los órganos deliberativos de las Naciones Unidas, constituye un lujo que no puede permitirse dada la urgencia de los problemas por solucionar. Las explicaciones teóricas preexisten, o bien es posible actuar sin ellas" (Wolfe, 1982). El autor afirma que este desdén frente a lo teórico ocurrió porque buena parte del público de la ONU está constituido por hacedores de política y opinión pública en general, quienes parecen no mostrarse interesados en estas cuestiones. 
Para Wolfe, la evasión del debate teórico en el marco institucional de las Naciones Unidas deja en evidencia varios asuntos. Por un lado, que en lugar del debate conceptual se abre paso a una suerte de "innovación" terminológica, es decir, el uso de términos sin ningún tipo de sustento ni debate, lo que de alguna manera oculta la falta de consenso en torno a las distintas concepciones existentes sobre la sociedad. Por otro lado, que la investigación aun en el marco institucional se encuentra atravesada por asuntos ideológicos, burocráticos, asuntos de las propias posiciones disciplinares, pero sobre todo, evidencia que, al tratarse de una actividad proveedora de empleo, "alienta a los técnicos a aplicar una mezcla juiciosa de espíritu de innovación y conformidad" (Wolfe,op.cit p.8). Reconocer este lugar no es un dato menor, pues lleva a la pregunta por las implicaciones de la producción de ideas y de conocimiento en un contexto de subordinación laboral y en un proceso de recepción de salario por el trabajo realizado, y además, tal como lo señala Estela Grassi “ciertos problemas del contenido conceptual y epistemológico del Trabajo Social no pueden pensarse aislados del contexto general de producción de las ideas en las materias que le son afines y de las condiciones y las formas institucionales de producción de conocimientos; más aún, de su inscripción en el proceso de producción de las interpretaciones políticas" (Grassi, 2007)

De otro lado, cabe advertir que la presencia de profesionales del Trabajo Social como parte de la burocracia de las Naciones Unidas se realizó desde dos lugares. El primero de ellos como parte del staff con responsabilidades individuales asumiendo diversos roles, el segundo, como se ha venido señalando, como miembros consultivos del ECOSOC. Así, se involucraron desde distintos lugares en las acciones de la entidad y sus agencias especializadas. A continuación se describen brevemente algunas de estas participaciones profesionales.

Si bien gran parte de las publicaciones de las Naciones Unidas son documentos oficiales y la autoría es institucional, en lo que respecta al Trabajo Social estas publicaciones han estado bajo la autoría y direccionamiento de profesionales del área. Así por ejemplo, el primer estudio internacional de formación para el Trabajo Social (1947-1950), fue realizado por la trabajadora social Katherine Kendall (1910-2010), que en ese momento era empleada de la oficina de asuntos sociales del ECOSOC. De acuerdo con las organizaciones de trabajadores sociales, los estudios de Kendall, fueron fundamentales para orientar la asistencia de servicios sociales y para la definición de la promoción de la educación del Trabajo Social desde la ONU (Kendall K. , 2008). 
En la UNICEF, fue el trabajador social Herman Stein (1917-2009) quien tuvo una destacada participación. No solo presentó un documento en el cual planteó directrices a los gobiernos para adelantar programas orientados a satisfacer las necesidades de los niños en el año 1964, sino que se desempeñó como asesor y consejero mayor. Adicionalmente participó de manera activa en la conferencia de ministros de bienestar social (1964) donde se discutió el papel del bienestar social en los programas de desarrollo, donde redactó muchos de los hallazgos y conclusiones (Kendall K. , 2005).

René Sand (1877-1953), por su parte siendo médico de origen belga, adelantó buena parte de su trabajo en coordinación con organizaciones y profesionales del Trabajo Social llegando incluso a ser miembro activo de las mismas. Tal fue su interés que tradujo la obra de Mary Richmond al francés, escribió el texto titulado "Tópicos, métodos y principios del Trabajo Social" (1919) y fue fundador del primer instituto de formación en Trabajo Social en Bélgica en 1919. Su vinculación y aporte al trabajo de las Naciones Unidas lo hizo por la vía de su participación activa en la creación de la Organización Mundial de la Salud, desde la cual abrió un nuevo eje de reflexión: la medicina social.

Finalmente Jan Floris de Jongh (1910-1988), además de haber presidido la Asociación Internacional de Escuelas de Trabajo Social en 1964 fue el primer director del Instituto de las Naciones Unidas de Investigación para el Desarrollo Social (UNRISD) en Ginebra, que fue creado durante la primera década del desarrollo en el que se pretendía ampliar el enfoque centrado en asuntos meramente económicos. Desde ese cargo se ocupó de establecer la relación entre Trabajo Social, planificación social y promover el Trabajo Social en África y en Asia (Riga, 2008).

Si bien la vinculación de un numeroso grupo de profesionales del Trabajo Social fue amplia, lo que quiero aquí es presentar un panorama general de su participación. Aunque la confianza que parece haber depositado la ONU en su trabajo es visible, en otros escenarios como el político y el académico en cambio al parecer dicha participación fue objeto de señalamientos, incluso al interior del propio Trabajo Social.

En efecto, en el escenario político por ejemplo, se les acusó de ser profesionales burgueses. Quizás una de las medidas más contundentes que se tomaron por asumirlos de esta manera ocurrió durante el periodo de intensificación de la GUERRA FRÍA en el que la formación en Trabajo Social fue eliminada 
7 El segundo en 1949 en Brasil, el tercero en Puerto Rico en 1957 y el cuarto en Costa Rica en 1961. Los encuentros panamericanos mediados por la OEA se realizaron hasta el año 1971, siendo el quinto en Lima (1965); el sexto en Caracas (1968) y el séptimo en Quito (1971). En adelante los congresos y seminarios se realizaron desde la autonomía de las organizaciones de trabajadores sociales en Latinoamérica.

8 Si bien este no fue e único seminario regional, es un referente importante por dos razones, la primera porque al ser el primer seminario regional marcó la pauta con la que se continuaron realizando los siguientes y la segunda porque fue la punta del "iceberg" que tomó la denominada "generación del 65" de trabajadores sociales del cono sur para problematizar las ideas que se estaban difundiendo en Latinoamérica sobre Trabajo Social y que posteriormente se derivó en la llamada reconceptualización. de los países comunistas (Watss, Elliot, \& Mayadas, 1995). En esta misma línea emergió en América Latina hacia las décadas del 60 y 70 del siglo XX, la llamada reconceptualización, como un movimiento al interior del propio Trabajo Social que se bien reivindicaba el lugar disciplinar, cuestionaba las prácticas profesionales ligadas a la caridad y al dominio institucional. En el escenario académico por su parte, al Trabajo Social se le señaló como una suerte de activismo con incipientes desarrollos teóricos y una pobre perspectiva crítica (Perelmiter, 2012).

¿Cómo fueron recibidas las ideas de Trabajo Social planteadas desde las Naciones Unidas en América Latina?

\section{La recepción de las ideas de trabajo social planteadas por las Naciones Unidas en América Latina}

En principio las referencias muestran que las orientaciones de la ONU llegaron a Latinoamérica a través de conferencias, encuentros y seminarios regionales organizados por la Unión Panamericana (que a partir de 1948 fue denominada Organización de Estados Americanos -OEA-) y si bien profesionales del trabajo social se articularon como parte del engranaje propuesto desde el norte global, su acción en este lado del mundo más que de agentes productores del discurso fue de reproductores del mismo. En efecto, en 1945 en Chile se llevó a cabo el Primer Congreso Panamericano de Servicio Social ${ }^{7}$, encaminado a revisar los avances en materia del Servicio Social en la región. También se adelantaron seminarios regionales entre 1950 y 1951 en Ecuador, El Salvador y Brasil abordando no solo diversas temáticas sino realizando un fuerte énfasis en el componente metodológico del trabajo social. Eran eventos organizados por las agencias especializadas de las Naciones Unidas.

Para 1957 en Uruguay, este organismo realizó un seminario regional sobre enseñanza del Servicio Social, en el que participaron expertos, observadores y representantes de diversos gobiernos latinoamericanos, muchos de los cuales no era profesionales del Trabajo Social ${ }^{8}$. El objetivo de dicho seminario era revisar si la formación en Servicio Social que se venía adelantando correspondía a las condiciones que el desarrollismo requería. Si bien el mayor número de ponencias de este evento fueron presentadas por profesionales de Trabajo Social latinoamericanos, puede advertirse que, lejos de asumir una postura crítica, estos trabajadores sociales lo que parecían hacer era una 
suerte de rendición de cuentas de lo que venían realizando en sus países a la luz de las orientaciones de la ONU.

De hecho, la relatora oficial del evento fue la asistente social chilena Valentina Maidagan de Ugarte, quien como funcionaria empleada de dicha entidad tenía la tarea de la difusión y asesoría en la creación de oferta de programas tanto de Trabajo Social como en formación posgradual, al mismo tiempo que de acciones de capacitación para auxiliares de Servicio Social en Latinoamérica. De la misma manera, fue la autora del "manual para el servicio social" publicado como texto de la ONU para Latinoamérica en el año 1963.

Los temas de debate en este seminario regional fueron entre otros, la edad necesaria para el ingreso en la formación en trabajo social, perfil de egresados, planes de estudio. Allí se definió la necesidad de ampliar la vinculación de hombres a los procesos formativos para que se desempeñaran principalmente en el área de desarrollo de la comunidad dado que en América Latina el trabajo social tuvo un marcado énfasis femenino (por lo menos así se promovió). Del mismo modo, se debatió en torno a contenidos teórico-prácticos que debían recibir los futuros profesionales y en ese aspecto, se afirmó que existía un excesivo número de asignaturas de formación teórica (Naciones Unidas, 1957, p.17). Lo cual evidencia que la orientación que la ONU hizo del Trabajo Social tuvo un marcado énfasis en lo práctico, lo que coincide con los planteamientos de Wolfe arriba señalados, en cuanto a que, en torno a lo social, no se lograron debates ni elecciones fundamentadas en las teorías existentes (Wolfe, 1982).

El seminario regional también incluyó una reflexión sobre la tarea de las ciencias sociales en la formación de Trabajo Social. Se planteó que si bien el trabajador social debía poseer conocimientos en dicha área, no debía alcanzar el mismo dominio, grado e intensidad que tenían quienes se formaban en ella, pues la función del trabajador social era "facilitar los cambios de actitud y comportamiento social exigidos por las reformas que se consideren deseables en la alimentación, en la higiene, en la conducta de la vida familiar y demás aspectos de la vida individual y colectiva" (Naciones Unidas, 1957), en otras palabras, la institución definió que fuese un profesional que actuara sobre la esfera subjetiva de tal manera que se dejaba en evidencia que el capitalismo no era solo un sistema económico, sino un sistema que prepara subjetividades sobre "la ilusión de que el mundo social puede funcionar con independencia de las epistemes locales y de la heterogeneidad" (Díaz, 2008). La tarea de profesionales de Trabajo Social era estimular y avivar el deseo de cambio. 
Pese a la aparente unidad entre los asistentes a este seminario, en el que participaron no solo representantes de diferentes Escuelas de Servicio Social latinoamericanas sino representantes gubernamentales (no trabajadores sociales pero sí funcionarios encargados de programas de bienestar social en sus respectivos países), fue el sociólogo Thomas Humprey Marshall (1957), representante de la UNESCO, quien se distanció planteando una ruptura con el papel asignado hasta el momento al Trabajo Social. En efecto, afirmó que el Trabajo Social desempeñaba funciones paliativas y desde su punto de vista debía trascender hacia la política social del país de cada profesional. Esta postura que cuestiona la tarea profesional, contrasta con las posiciones de trabajadores sociales, quienes en el mismo evento plantearon que "el trabajo en los programas de bienestar requiere gran abnegación y una actitud de sencillez de parte de los profesionales que trabajan en ellos" (Naciones Unidas, 1957). De esta manera las Naciones Unidas fueron construyendo su propio perfil del trabajador social: un técnico, encargado de la búsqueda de soluciones a los problemas sociales de manera coordinada con instituciones y adicionalmente, ingenioso, creativo, neutral; cualidades todas ellas que no requerían mayor fundamentación teórica pero sí destreza metodológica para la intervención social. Estimular el cambio de actitudes en las poblaciones parecía ser la tarea central que se les había confiado a estos profesionales.

A pesar de que la confianza en que el cambio social podía ser burocráticamente inducido, en el año 1964 la ONU comenzaba a tener dudas sobre si existía una fórmula que pudiera aplicarse a todos los países de manera práctica "Hemos traído un conjunto de principios y orientaciones que a nuestro juicio han servido en ciertas situaciones y cuya validez en otros casos debe al parecer comprobarse" (Consejo Económico y Social, 1964, p. 6). De hecho, este fue el contexto para introducir el Desarrollo de la Comunidad como alternativa "innovadora", al punto que se llegó a plantear que "el verdadero problema que tiene que resolver el desarrollo de la comunidad consiste en encontrar técnicas para motivar a la población a fin de que haga lo que es preciso hacer en una forma que aumente su confianza en sí misma y su capacidad de planificación" (Consejo Económico y Social, 1964)

Habría que señalar la influencia en la introducción de los principios del Desarrollo de la Comunidad en Latinoamérica, que tuvo la Unión Panamericana en cabeza de la asistente social de EEUU Caroline Ware. Ella fue la encargada de realizar capacitaciones en diversos países, su obra fue traducida al español y ampliamente difundida como manuales. Algunos 
documentos incluso la señalan como formadora de generaciones de asistentes sociales latinoamericanos ${ }^{9}$. Dentro de los profesionales locales se destaca la activa participación de la Señora Valentina Maidagan de Ugarte, asistente social chilena quien tuvo a cargo el proceso de reformas a las Escuelas de Servicio Social argentinas; Lina Vera de Vieira también chilena quien tuvo bajo su responsabilidad el documento de la "Conferencia latinoamericana sobre la infancia y la juventud en el desarrollo nacional" de la ONU en el año 1965 y Carmelina Capó, asistente social puertorriqueña quien encabezó una de las misiones de la UNESCO en Colombia y publicó el texto "Plan de vida familiar en Vianí" en el año 1951.

Para la década del 60 del siglo XX, estas ideas comenzaron a generar resistencias en algunos sectores del colectivo profesional latinoamericano. En efecto, no solo se manifestaron abiertamente en contra planteando rupturas con las funciones asignadas y asumidas, sino que también tomaron distancia con las conclusiones del tercer estudio internacional que diseñó programas de formación en Trabajo Social para América Latina (Kruse, 1986). Particularmente, emergió un colectivo en el cono sur autodenominado la "generación del 65" cuyas reflexiones derivaron en lo que posteriormente se conoció al interior del Trabajo Social latinoamericano como movimiento de reconceptualización, no solo oponiéndose a las recomendaciones de la ONU sino que también negaban el "diagnóstico" que la asistente social chilena Maidagán Ugarte había realizado, en el cual afirmaba que la formación en Servicio Social en Argentina tenía por lo menos treinta años de atraso.

La generación del 65 se propuso entonces cuestionar no solo los lineamientos de las agencias internacionales sino realizar una serie de propuestas que incluían la construcción de un nuevo plan de estudios, el cual se puso en marcha en el Uruguay en la Universidad de la República. Lo particular de este plan de estudios, era que por primera vez se establecía distancia con los consejos y lineamientos de la ONU y se estimuló la creación de publicaciones propias, encaminadas a pensar en un trabajo social latinoamericano. Así, se destacaron revistas como "Hoy en el servicio social" (1964-1977) y "Selecciones de servicio social" (1968-1978); de la misma manera se organizaron seminarios de Trabajo Social dinamizados desde los propios colectivos y escuelas y no desde los organismos internacionales como se venía haciendo.

Fue así como en el año 1965 se organizó el primer seminario autónomo y por fuera de las agencias internacionales de Servicio Social latinoamericano en el

9 Bonfiglio, op cit, señala que como experta tuvo un destacado papel en la divulgación de los planteamientos del Servicio Social promovidos por la Unión Panamericana en toda Latinoamérica, particularmente en lo referente a la organización y desarrollo de la comunidad. 
Brasil y en el mismo año en Lima se adelantó el Seminario Latinoamericano de Escuelas de Servicio Social del cual derivó la Asociación Latinoamericana de Escuelas de Servicio Social -ALAESS que luego, en 1977, fue ALAETS-.

Dentro de las primeras preocupaciones estaba lo que leían como ausencia de un marco teórico propio. Por ello definieron que sería el materialismo histórico el que en adelante adoptarían para lograr una amplia comprensión de la sociedad. Del mismo modo, se preguntaban por la cientificidad, ninguno de estos debates hacía parte de la agenda en los seminarios panamericanos ni regionales previos, pues el énfasis estaba puesto en el método (Kruse, 1969).

A este brote de perspectiva crítica, Alayón (1971) le denominó cambioresistencias, entendiendo que no se trataba de resistencias al cambio, sino un cambio visto de acuerdo a los contextos latinoamericanos. Dentro de los aspectos que el colectivo cuestionaba a los organismos internacionales eran la presencia de textos escritos en inglés, la neutralidad profesional que se le otorgaba al ejercicio del trabajo social y el traslado de métodos que no consideraban las realidades locales. En este orden de ideas, se trató de investigar, teorizar, enseñar y ensayar un Servicio Social genuinamente latinoamericano (Alayón, Barreix, \& Cassineri, 1971; 58).

El movimiento de reconceptualización fue la expresión del espíritu de la época en la década del 60 en Latinoamérica. En efecto, la preocupación por el ser latinoamericano rondaba muchos escenarios, al igual que la idea de la militancia y de pensar el cambio desde lo estructural en una perspectiva que acogía buena parte de los postulados marxistas y estimulaba el compromiso con la sociedad. Así por ejemplo, en ese momento la iglesia católica planteó una iglesia latinoamericana comprometida con los pobres, inspirada en el documento de Medellín (1968) bajo las premisas de la Teología de la Liberación, un ejemplo claro de militancia en este marco, lo representó Camilo Torres -sociólogo, sacerdote- quien decidió involucrarse en la lucha armada en Colombia; la revolución cubana en la que participó activamente el "Che" Guevara. Guevara, tuvo la oportunidad de asistir a una de las conferencias de las Naciones Unidas y ser escuchado en Uruguay en el año 1961; Paulo Freire por su parte desafió el tipo de educación que se pretendía y propuso una educación liberadora, mientras Enrique Dussel se planteaba una filosofía de la liberación; también Orlando Fals Borda cuestionó las maneras y los sentidos de la investigación social sociológica; mientras los economistas declaraban que "debían ser mucho más que técnicos, que la solidaridad obrera y campesina 
debía ser apoyada y que todas las industrias básicas y de servicios públicos, debían ser nacionalizados " (Babb, 1991, p. 677). Todas estas propuestas consideraban que investigar y actuar eran parte de un mismo proceso y en ese orden, proponían auscultar las raíces latinoamericanas, la búsqueda de la relación teoría y práctica con un claro compromiso frente al cambio social. Todo este "espíritu de época" permeó al Trabajo Social de tal manera que muchas de las discusiones se incorporaron como parte de la reflexión profesional de ese momento.

Pese a estas tensiones y distanciamientos con las agencias internacionales, encabezadas por las Naciones Unidas, tales agencias continúan siendo fuente de empleo profesional. Así por ejemplo, Ezequiel Ander Egg, sociólogo destacado editor de textos en Trabajo Social, fue consultor de las Naciones Unidas en planificación nacional y local y de la UNESCO en política cultural y animación sociocultural; de la misma manera consultor de la OEA en el tema de desarrollo de la comunidad. Boris Lima, trabajador social venezolano y representante del movimiento de reconceptualización, fue consultor de la División de Desarrollo de la CEPAL en 1982; Norberto Alayón, trabajador social argentino, hizo parte del movimiento de la generación del 65, estuvo vinculado como parte del equipo de la Campaña Mundial contra el Hambre promovida por la FAO entre 1966-1967; mientras María Lorena Molina, trabajadora social costarricense estuvo vinculada a través del Ministerio de la Presidencia de Costa Rica a la ACNUR-ONU.

Del mismo modo ocurre que las organizaciones internacionales de trabajadores sociales siguen estando activas en las Naciones Unidas y en el día mundial del trabajo social plantean, diseñan y ratifican la necesidad de una agenda global de Trabajo Social. Así mismo, este organismo continúa publicando documentos de formación en Trabajo Social en el que han participado de manera directa organizaciones profesionales ${ }^{1}$.

\section{CONCLUSIONES}

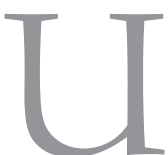

- n fenómeno particular que parece advertirse en la participación directa de trabajadores sociales y organizaciones de Trabajo Social - en las Naciones Unidas tiene que ver con el desplazamiento de los saberes generados en el Trabajo Social que fueron puestos en circulación y apropiados como saberes técnicos institucionales, globales, homogéneos y "aplicables" para cualquier contexto. En efecto, los denominados por el trabajo social norteamericano como métodos de la profesión tales como
10 Pueden mencionarse entre otros: - Serie de Capacitación Profesional No 1, Derechos Humanos y Trabajo Social. Manual para Escuelas de Servicio Social y Trabajadores Sociales Profesionales. Naciones Unidas Nueva York y Ginebra 1995. Documento elaborado con la colaboración de la Federación Internacional de Trabajadores Sociales.

-Social Work and the Rights of the Child. A Professional Training Manual on the UN Convention. International federation social workers A Professional Training Manual, on the UN Convention. 2002.

- Guidance and Counselling for School-age Girls in Africa (UNESCO) module 3: social work 2002.

En el cual se afirma que se trata de un paquete de capacitación sobre orientación y asesoramiento preparado por especialistas de varios países africanos, especialmente maestros considerando que en ocasiones necesitan herramientas para desempeñarse como trabajadores sociales en la escuela y en la comunidad a la que pertenecen. 
caso, grupo y comunidad con el proceso de modernización para el desarrollo fueron exportados como técnicas en manuales "desprofesionalizados" con la idea de que cualquier persona pudiese al revisarlos, llevarlos a cabo desde cualquier lugar y en cualquier momento. Del mismo modo, siguiendo los patrones de saber creados en el norte, principalmente Francia, Estados Unidos e Inglaterra, publicaciones, becas y profesionales locales operaban como "correas de transmisión" en un contexto de circulación internacional de ideas sobre lo social.

Así, la intervención sobre la cuestión social pareciera que emerge, siguiendo los planteamientos de Foucault, como un umbral en el arte de gobernar poblaciones, esta vez no desde un territorio delimitado bajo la soberanía del Estado, ni desde un gobernante local sino desde un aparato compuesto por una interrelación de Estados dispuestos a gobernar sus poblaciones que son el blanco de intervenciones de todo tipo. Así, el desarrollo de aparatos y saberes a través de un conjunto de instituciones, procedimientos, reflexiones, análisis, cálculos, tácticas, permite la extensión de formas de poder sobre los otros, en los que tanto el Trabajo Social como las ciencias sociales han tenido un papel fundamental.

En todo este proceso, se puede notar que al Trabajo Social se le asignó la tarea de ser un apoyo intergubernamental para afianzar el capitalismo realizando un trabajo sobre la subjetividad, pues el modo de producción capitalista necesita un modo de vida capitalista (Díaz, 2008). Así mismo, en tanto el Trabajo Social entró a compartir la reorganización de las ciencias sociales propuesta desde las Naciones Unidas, asumió la tarea de contribuir a la reorganización de las relaciones de fuerza en el sistema mundo (Díaz, 2008); por ello, tanto las ciencias sociales como el trabajo social de la ONU, concentraron todos sus esfuerzos en tecnificar modos de intervención sobre lo social en función del modo de vida capitalista, asunto que es necesario continuar explorando.

Lo que parece advertirse aquí es la relación saber-poder. En efecto, la propuesta de intervención de las Naciones Unidas en el marco del desarrollo, exigió una nueva organización de saberes al punto que las ciencias sociales se perfeccionaron como paquetes técnicos de investigación (Díaz, 2008). Así, mientras las misiones de expertos se encargaron de visibilizar los puntos de intervención, los pueblos debieron gestionar por sí mismos su ingreso a lo que el desarrollo exigió. 
De este modo, el conocimiento que se produce en un contexto de institucionalidad interestatal como lo representa las Naciones Unidas, es un tipo de conocimiento sobre la población y sobre su territorio mediado por la aspiración al cálculo y la previsión que responde a demandas institucionales. Cabe destacar que de alguna manera tales demandas generan un proceso de normalización del poder de los "expertos" organizados en cuerpos técnicos de profesionales, así, "las profesiones emergen, se desarrollan (y también declinan) en el dinámico contexto de la competencia con otras profesiones y con grupos al interior de la misma profesión. Todas las profesiones tienen que asegurar que sus esfuerzos por monopolizar la oferta de conocimientos técnicos son percibidos como necesarios, beneficiosos y, por lo tanto, legítimos" (Montecinos, 1997), en otras palabras, todas las profesiones deben mostrar que su saber es necesario.

\section{REFERENCIAS BIBLIOGRÁFICAS}

ALAYÓN, Norberto; Barreix, Juan \& Cassineri, Ethel. (1971). ABC del Trabajo Social Latinoamericano. Buenos Aires: Ecro.

ANDER Egg, Ezequiel. (2000). Metodología y práctica del desarrollo comunitario. Buenos Aires: Lumen.

BABB, Sara. (1991). Los profesionistas en el gobierno y el problema d ela tecnocracia: el caso de los economistas en México. En Estudios Sociológicos. México. Colegio Mayor de México.Vol.16, No. 48 (septiembre-diciembre) pág. 661-688.

BONFIGLIO, Giovanni. (1982). Desarrollo de la comunidad y trabajo social. Lima: Ediciones Celats. CONSEJO ECONÓMICO Y SOCIAL. (1964). Seminario regional latinoamericano sobre el papel del desarrollo de la comunidad en la aceleracion del desarrollo económico y social. Dirección de Asuntos Sociales. Nueva York: ONU.

CRONIN, Michael; SAKINA Mama; ROBIN, Mbugua \& MOURAVIEFF-APOSTOL, Elle. (2006). Social Work and the United Nations. In N. (. Hall, Social Work: Making a world of difference. Social Work around the world IV in the years of IFSW, 50 th jubilee. (pp. 209-224). Berne, Switzerland: FITS.

DíAZ DE MIRANDA, María. (1961). El servicio social como un proceso educativo. Cuarto Congreso Panamericano de Servicio Social (p. 15). San José, Costa Rica : 19-25 noviembre.

DÍAZ, D. (2008). Raza, pueblo y pobres. Las tres estrategias biopolíticas del siglo XX en Colombia (1873-1962). In S. Castro Gómez, \& E. (. Restrepo, Genealogías de colombianidad. formaciones discursivas y tecnologías de gobierno en los siglos XIX y XX. (pp. 42-69). Bogotá: Pontificia Universidad Javeriana. 
EILERS, K. (23 de Julio de 2015). René Sand (1877-1953) and His Contribution to International Social Work, IASSW-President 1946 - 1953. From Social work and society: http://www.socwork. net/sws/article/view/124/540

GRASSI, Estela. (2007). Problemas de realismo y teoricismo en la investigación social y en el Trabajo Social. Katalysis, V 10 , 26-36.

GROFOSGUEL, R. (2006). La descolonización de la economía política y los estudios postcoloniales: transmodernidad, pensamiento fronterizo y colonialidad global. Tabula rasa , 17-46.

KENDALL, Katherine. (1 de junio de 2005). Herman D. Stein (USA), President 1968 - 1976. Social Work and Society , 273-281.

KENDALL, Katherine. (15 de agosto de 2008). Annual Social Work Day. From Council of social work education: http://www.cswe.org/File.aspx?id=25145

KRUSE, Herman. (1986). Filosofía del siglo XX y servicio social. . Buenos Aires: Hvmanitas.

LYONS, K., Hokenstad, T., Pawar, M., Huegler, N., \& Hall, N. (2012). The Sage handbook of international social work. London: Sage, publications.

LÓPEZ, M. (1981). Intervención imperialista en las políticas de desarrollo y bienestar social. In M. E. Martínez, L. Miriam, M. Saboyá, R. R. Elena, \& A. Poveda, Historia del Trabajo Social en Colombia (pp. 71-105). Bogotá: Tecnilibros.

MASTRANGelo DE PAMPHilis, R. (2003). Acerca del objeto del traba jo social. Buenos Aires: Lumen Humanitas.

MIGNOLO, W. (2003). Historias locales/ Diseños Globales. Colonialidad, conocimientos subalternos, y pensamiento fronterizo. Madrid: Akal.

MOLINA, Lorena et al. (20 de julio de 2015). CONTRIBUCIONES AL DEBATE SOBRE EL FUTURO DE ALAETS/CELATS. From Universidad de Costa Rica: http://www.ts.ucr.ac.cr/binarios/congresos/ reg/slets/slets-018-015.pdf

MONTECINOS, V. (1997). Los economistas y las élites políticas en América Latina. Revista Estudios Internacionales, Universidad de Chile. , 351-375.

NACIONES UNIDAS. (1957). Seminario sobre enseñanza del servicio social en América Latina. (p. 284). Montevideo: Naciones Unidas.

ORGANIZACIÓN DE ESTADOS AMERICANOS (OEA). (1956). VIII Curso Internacional de Reforma Agraria . Ubicación de la antropología en un programa de desarrollo . Lima: OEA.

ORGANIZACIÓN DE LAS NACIONES UNIDAS PARA LA EDUCACIÓN, LA CIENCIA Y LA CULTURA -UNESCO-. (1949). El correo de la Unesco . Vol II , No. 8. 
ORGANIZACIÓN MUNDIAL DE LA SALUD. (1952). Health Education in Upper Egypt. Chronicle of the world health organization, 355-357.

PERELMITER, Luisina. (2012). Saber asistir: Técnica, política y sentimiento en la asistencia estatal Argentina (2003-2008). In S. Moressi, \& G. Vommaro, Saber lo que se hace, expertos y política en Argentina. (p. 399). Buenos Aires: Prometeo.

POBLETE TRONCOSO, M. (1958). La comunidad internacional contemporánea: relaciones y organismos internacionales. Santiago de Chile: Jurídica.

RIGA, Albert. (25 de septiembre de 2008). Jan Floris de Jongh (Netherlands), President $1954-$ 1961. Social Work and Society. International online journal. , 179-185.

SCOTT, J. (2001). Experiencia. La ventana , 42-73.

UNESCO. (1985). Unesco en su 40 aniversario. Madrid : Graficum.

WALLERSTEIN, Inmanuel. (. (2006). Abrir las ciencias sociales. Buenos Aires: Siglo XXI.

WATSS, Thomas., ELLIOT; DDoreen \& Mayadas, N. (1995). International Hand book. Social Work education. United States: Congress Library.

WOLFE, Marshall. (1982). El desarrollo esquivo. La búsqueda de un enfoque unificado para el análisis y la planificación del desarrollo. Revista de la Cepal , 7-50. 